\title{
HALO GAS CROSS SECTIONS AND COVERING FRACTIONS OF Mg II ABSORPTION SELECTED GALAXIES
}

\author{
Glenn G. KacprzaK ${ }^{1}$, Christopher W. Churchill ${ }^{1}$, Charles C. Steidel ${ }^{2}$, And Michael T. Murphy M $^{3,4}$ \\ ${ }^{1}$ New Mexico State University, Las Cruces, NM 88003, USA; glennk@nmsu.edu, cwc@nmsu.edu \\ 2 Caltech, Pasadena, CA 91125, USA; ccs@astro.caltech.edu \\ ${ }^{3}$ Institute of Astronomy, Cambridge CB3 OHA, UK \\ ${ }^{4}$ Swinburne University of Technology, Hawthorn, Victoria 3122, Australia; mmurphy@astro.swin.edu.au \\ Received 2007 August 29; accepted 2007 October 29; published 2008 February 5
}

\begin{abstract}
We examine halo gas cross sections and covering fractions, $f_{c}$, of intermediate-redshift $\mathrm{Mg}$ II absorption selected galaxies. We computed statistical absorber halo radii, $R_{\mathrm{x}}$, using current values of $d N / d z$ and Schechter luminosity function parameters, and have compared these values to the distribution of impact parameters and luminosities from a sample of 37 galaxies. For equivalent widths $W_{r}(2796) \geqslant 0.3 \AA$, we find $43 \leqslant R_{\mathrm{x}} \leqslant 88 \mathrm{kpc}$, depending on the lower luminosity cutoff and the slope, $\beta$, of the Holmberg-like luminosity scaling, $R \propto L^{\beta}$. The observed distribution of impact parameters, $D$, are such that several absorbing galaxies lie at $D>R_{\mathrm{x}}$ and several non-absorbing galaxies lie at $D<R_{\mathrm{x}}$. We deduced that $f_{c}$ must be less than unity and obtain a mean of $\left\langle f_{c}\right\rangle \sim 0.5$ for our sample. Moreover, the data suggest that halo radii of $\mathrm{Mg}$ II absorbing galaxies do not follow a luminosity scaling with $\beta$ in the range of $0.2-0.28$, if $f_{c}=1$ as previously reported. However, provided $f_{c} \sim 0.5$, we find that halo radii can remain consistent with a Holmberg-like luminosity relation with $\beta \simeq 0.2$ and $R_{*}=R_{\mathrm{x}} / \sqrt{f_{c}} \sim 110 \mathrm{kpc}$. No luminosity scaling $(\beta=0)$ is also consistent with the observed distribution of impact parameters if $f_{c} \leqslant 0.37$. The data support a scenario in which gaseous halos are patchy and likely have non-symmetric geometric distributions about the galaxies. We suggest that halo gas distributions may not be governed primarily by galaxy mass/luminosity but also by stochastic processes local to the galaxy.
\end{abstract}

Key words: galaxies: halos - galaxies: ISM - quasars: absorption lines

\section{INTRODUCTION}

Understanding galaxy formation and evolution is one of the most important topics of modern astronomy. The extended distribution of baryonic gas surrounding galaxies holds great potential for constraining theories of their formation. However, the sizes of gaseous galaxy halos along with the distribution of gas within are not well understood. Numerical models have been able to synthesize the formation and evolution of large scale structures; however, there are unresolved issues regarding the evolution of individual galaxies and halos. The halo baryonfraction problem (e.g., Mo \& Mao 2002) and the rapid cooling of gas (e.g., White \& Rees 1978) result in galaxy halos which have little or no gas soon after they form. These effects are not seen in the observable universe since there is an abundance of galaxies where gas has been detected in halos via quasar absorption lines.

From an observational standpoint, quasar absorption lines provide a unique means of probing the extent and abundance of halo gas. Although quasar absorption line observations to date are sufficient to recognize the aforementioned problems, they are lacking the details required to statistically constrain the distribution of the baryonic gas in the halos of simulated galaxies. Cross-correlations between absorbers and galaxies hold the promise to yield useful information on cloud sizes and halo gas covering fractions. First steps toward incorporating multiphase gas in semi-analytical models and numerical simulations suggest that warm gas in halos extends out to galactocentric distances of $\sim 150 \mathrm{kpc}$ with cloud covering fractions of $\sim 0.25-0.6$ (Maller \& Bullock 2004; Kaufmann et al. 2006).

The association of $\mathrm{Mg}$ II $\lambda \lambda 2796,2803$ doublet absorption in quasar spectra with normal, bright, field galaxies has been firmly established (e.g., Bergeron \& Boissé 1991; Steidel et al. 1994; Churchill et al. 2005). In an effort to understand halo sizes and gas distributions, Steidel (1995, hereafter S95) searched for foreground galaxies associated with $\mathrm{Mg}$ II absorption within $\sim 10^{\prime \prime}(\sim 65 \mathrm{kpc}$ for $z=0.5)$ of quasars. ${ }^{5}$ The sample consisted of 53 absorbing and 14 non-absorbing galaxies with a Mg II $\lambda 2796$ equivalent width sensitivity limit of $W_{r}(2796)>$ $0.3 \AA$ A. S95 directly fitted the data by assuming a Holmberg-like luminosity scaling,

$$
R(L)=R_{*}\left(\frac{L}{L^{*}}\right)^{\beta} \mathrm{kpc},
$$

and minimizing the number of non-absorbing and absorbing galaxies above and below the $R(L)$ relation. The best fit obtained clearly showed that absorbing and non-absorbing galaxies could be separated and that the halo radii $R\left(L_{K}\right)$ and $R\left(L_{B}\right)$ scale with luminosity with $\beta=0.15$ and $\beta=0.2$, respectively, where an $L_{B}^{*}$ galaxy has a gas halo cross section of $R_{*}=$ $55 \mathrm{kpc}$. Furthermore, since almost none of the absorbing galaxies were observed above the $R(L)$ boundary and that almost none of the non-absorbing galaxies were observed below the $R(L)$ boundary, S95 inferred that all $L>0.05 L^{*}$ galaxies are hosts to $\mathrm{Mg}$ II absorbing gas halos characterized by a covering fraction of unity and a spherical geometry which truncates at $R(L)$. Examination of this now "standard model" has been the subject of several theoretical studies (e.g., Charlton \& Churchill 1996; Mo \& Miralda-Escude 1996; Lin \& Zou 2001).

Guillemin \& Bergeron (1997) determined a steeper value of $\beta=0.28$ for the $B$-band luminosity obtained from a best fit to the upper envelope of the distribution of impact parameters of 26 absorbing galaxies. They found $R_{*}=67 \mathrm{kpc}$.

\footnotetext{
5 Throughout we adopt a $h=0.70, \Omega_{\mathrm{M}}=0.3, \Omega_{\Lambda}=0.7$ cosmology. All quoted physical quantities from previously published works have been converted to this cosmology.
} 
Using a reverse approach of establishing foreground galaxy redshifts and then searching for $\mathrm{Mg}$ II absorption in the spectra of background quasars yields results inconsistent with a covering fraction of unity. For example, Bowen et al. (1995) identified 17 low-redshift galaxies with background quasar probing an impact parameter range between 3 and $162 \mathrm{kpc}$. Galaxies that were probed at impact parameters greater than $13 \mathrm{kpc}$ had no absorption in the halo $\left(W_{r}(2796) \geqslant 0.40-0.9 \AA\right)$; however, four of the six galaxies within $13 \mathrm{kpc}$ of the halo produced $\mathrm{Mg}$ II absorption. For intermediate-redshift galaxies, Bechtold $\&$ Ellingson (1992) reported a covering fraction $f_{c} \simeq 0.25$ for $W_{r}(2796) \geqslant 0.26 \AA$ for eight galaxies with $D \leqslant 85 \mathrm{kpc}$. Also, Tripp \& Bowen (2005) reported $f_{c} \sim 0.5$ for $W_{r}(2796) \geqslant$ $0.15 \AA$ for $\sim 20$ galaxies with $D \leqslant 50 \mathrm{kpc}$. These results are also consistent with the findings of Churchill et al. (2005) who reported very weak $\mathrm{Mg}$ II absorption, $W_{r}(2796)<0.3 \AA$, well inside the $R(L)$ boundary of bright galaxies; these galaxies would be classified as "non-absorbers" in previous surveys. They also report $W_{r}(2796)>1 \AA$ absorption out to $\simeq 2 R(L)$. All these results suggest that there are departures from the standard model, that the covering fraction of $\mathrm{Mg}$ II-absorbing gas is less than unity, and that the halo sizes and the distribution of the gas appear to diverge from the $R(L)$ relation with spherical geometry.

Another approach to understanding halo sizes and gas distributions is to determine the statistical properties of $\mathrm{Mg}$ II absorbing gas and then compute the statistical cross section from the redshift path density, $d N / d z$ (see Lanzetta et al. 1995). The downfall of this method is that a galaxy luminosity function must be adopted in order to estimate $R_{*}$. Nestor et al. (2005) acquired a sample of over $1300 \mathrm{Mg}$ II absorption systems, with $W_{r}(2796) \geqslant 0.3 \AA$ from the Sloan Digital Sky Survey (SDSS). Using the $K$-band Holmberg-like luminosity scaling and luminosity function of MUNICS (Drory et al. 2003), Nestor et al. computed $R_{*}=60-100 \mathrm{kpc}$ for adopted minimum luminosity cutoffs of $L_{\min }=0.001-0.25 L^{*}$. They found no redshift evolution of $R_{*}$ over the explored range of $0.3 \leqslant z \leqslant 1.2$.

Zibetti et al. (2007) studied the statistical photometric properties of $\sim 2800 \mathrm{Mg}$ II absorbers in quasar fields imaged with SDSS. Using the method of image stacking, they detected lowlevel surface brightness (SB) azimuthally about the quasar. The SB profiles follow a decreasing power law with projected distance away from the quasar out to $100-200 \mathrm{kpc}$. These results imply that absorption selected galaxies may reside out to projected distances of $200 \mathrm{kpc}$. However, it is worth noting that the extended light profiles may be an artifact of clustering of galaxies. Cluster companions of the $\mathrm{Mg}$ II absorbing galaxies could extend the observed light profile over hundreds of stacked images. Thus, one would infer that $\mathrm{Mg}$ II absorbing galaxies are present at larger impact parameters than would be found in direct observation of individual galaxies.

Motivated by recent expectations from simulations that halo gas is dynamically complex and sensitive to the physics of galaxy formation, we investigate the standard halo model of Mg II absorbers. We also aim to provide updated constraints on $f_{c}$ and $\beta$ for galaxy formation simulations. In this paper, we demonstrate that $f_{c}<1$ and question the validity of the Holmberg-like luminosity scaling (Equation (1)). Using high resolution quasar spectra, we explore $\mathrm{Mg}$ II absorption strengths to an order of magnitude more sensitive than previous surveys which allow us to re-identify non-absorbing galaxies as "weak" absorbing galaxies. In Section 2, we describe our sample and analysis. In Section 3, we present new calculations of the statistical absorber radius computed using the statistically measured absorption path density $d N / d z$ and the Schechter luminosity function. We then compare these values to the empirical results of $\mathrm{S} 95$ and to a sample of known $\mathrm{Mg}$ II absorption selected galaxies with measured luminosities and impact parameters. We also examine how individual halos behave with respect to the statistical halo. In Section 4, we discuss the properties and distribution of gas in halos. Our concluding remarks are in Section 5.

\section{DATA AND ANALYSIS}

We have constructed a sample of 37 galaxies $(0.3<z<1.0)$, with spectroscopically confirmed redshifts, selected by the presence of Mg II absorption in quasar spectra. The absorption properties were measured from HIRES/Keck (Vogt et al. 1994) and UVES/VLT (Dekker et al. 2000) spectra. The Mg II $\lambda 2796$ profiles have been presented in Churchill et al. (2005), where the detection limit is $W_{r}(2796) \geqslant 0.02 \AA(5 \sigma)$. Galaxy properties were measured from F702W or F814W WFPC-2/HST images of the quasar fields. Images of the galaxies, along with further details of the sample selection, data, and data analysis, can be found in Kacprzak et al. (2007a).

Galaxy absolute magnitudes, $M_{B}$, were determined from the $k$-corrected observed $m_{\mathrm{F} 702 \mathrm{~W}}$ or $m_{\mathrm{F} 814 \mathrm{~W}}$ adopted from Kacprzak et al. (2007a). The $k$-corrections were computed using the formalism of Kim et al. (1996) based upon the spectral energy distribution (SED) templates of Kinney et al. (1996). The adopted SED for each galaxy was based upon its rest-frame $B-K$ color (SDP94). For galaxies with no color information, we adopted a Sb SED which is consistent with average color of Mg II absorbing galaxies (SDP94; Zibetti et al. 2007). Our $k$-corrections are consistent with those from the literature (Kim et al. 1996; Fukugita et al. 1995). B-band luminosities were computed using the DEEP2 optimal $M_{B}^{*}$ of Faber et al. (2007, Table 2) in the redshift bin appropriate for each galaxy. $M_{B}^{*}$ ranges from $-21.07(\langle z\rangle=0.3)$ to $-21.54(\langle z\rangle=1.1)$.

We compute the halo gas cross section determined from the redshift path density,

$$
\frac{d N}{d z}=\pi R_{\mathrm{x}}^{2} \cdot \Phi^{*} \Gamma(x, y) \cdot \frac{c}{H_{0}} \frac{(1+z)^{2}}{\sqrt{\Omega_{m}(1+z)^{3}+\Omega_{\Lambda}}},
$$

where $R_{\mathrm{x}}$ is the statistical absorber radius for an $L^{*}$ galaxy, and $\Phi^{*}$ is the number density of $L^{*}$ galaxies. $R_{x}^{2}=f_{c} R_{*}^{2}$, where $R_{*}$ is the covering fraction corrected absorbing halo radius. Note here that we make a distinction between $R_{\mathrm{x}}$, which is derived from the redshift path density, and $R_{*}$, which is a physical cross section of the absorbing gas accounting for the covering fraction. $\Gamma(x, y)$ is the incomplete Gamma function in which $x=2 \beta-\alpha+1$, where $\alpha$ is the faint-end slope of the Schechter galaxy luminosity function and $\beta$ parameterizes a Holmberg-like luminosity scaling of Equation (1). The parameter $y=L_{\min } / L^{*}$, where $L_{\min }$ is the minimum luminosity of galaxies contributing to absorption. The influence of $y$ on the value of $R_{\mathrm{x}}$ becomes relatively more important as $\beta \rightarrow 0$.

We present our sample in Figure 1(a), plotting $W_{r}(2796)$ versus $L_{B} / L_{B}^{*}$. The solid points have $W_{r}(2796) \geqslant 0.3 \AA$ and the open points are weak systems (Churchill et al. 1999), having $W_{r}(2796)<0.3 \AA$, and would have been classified as nonabsorbing galaxies in previous surveys (e.g., SDP94; Guillemin \& Bergeron 1997). Since $R_{\mathrm{x}}$ is computed using $d N / d z$ which is determined for systems with $W_{r}(2796) \geqslant 0.3 \AA$, we must 

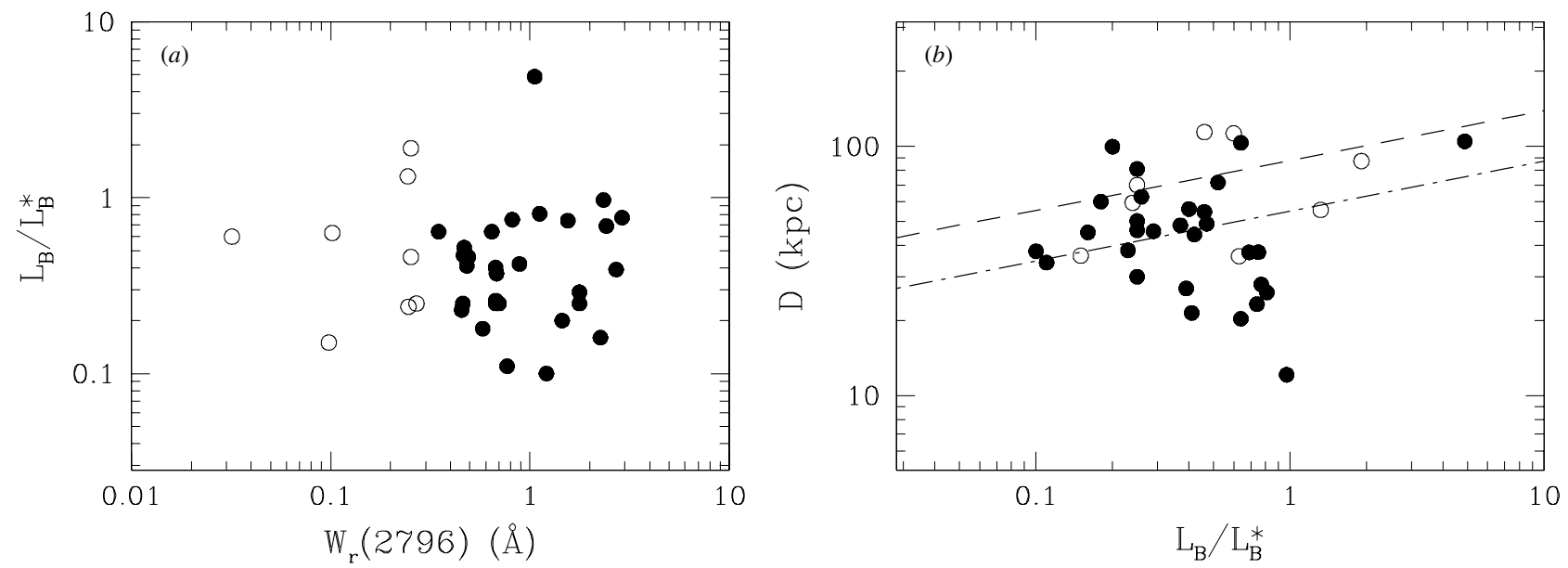

Figure 1. (a) $W_{r}$ (2796) versus $L_{B} / L_{B}^{*}$. Filled circles have $W_{r}(2796) \geqslant 0.3 \AA$ and the open circles have $W_{r}(2796)<0.3 \AA$. (b) The impact parameter, $D$, versus $L_{B} / L_{B}^{*}$. The dash-dot line is the halo luminosity scaling given by Equation (1) for the results of $\mathrm{S} 95\left(R_{*}=55 \mathrm{kpc}, f_{c}=1, \beta=0.2\right)$. The dash-dash line is the halo luminosity scaling given by Equation (1) for our result $\left(R_{*}=88 \mathrm{kpc}\right)$, assuming $f_{c}=1, \beta=0.2$.

consider these "weak" systems as "non-absorbing" galaxies in order to be consistent with our comparisons for the remainder of this paper. In Figure 1(a) note that both absorbing and nonabsorbing galaxies span the same luminosity range.

\section{RESULTS}

Applying Equation (2), we computed the statistical absorption radius, $R_{\mathrm{x}}$, for $W_{r}(2796) \geqslant 0.3 \AA$ employing the most current Schechter luminosity function parameters and absorber redshift path density. We adopted $d N / d z=0.8$ (Nestor et al. 2005), $\alpha=1.3$, and $\Phi_{*}=3.14 \times 10^{-3} \mathrm{Gal} \mathrm{Mpc}^{-3}$ (Faber et al. 2007) for the $\langle z\rangle=0.5$ redshift bin, where the mean redshift of our sample is 0.58 . Since the luminosity scaling is not necessarily constrained by our sample, we consider both $\beta=0.2$ and $\beta=0$ (i.e., no scaling) for $y=0.05$ and $y=0.01$. We obtained

$$
R_{\mathrm{x}}=\sqrt{f_{c}} R_{*}= \begin{cases}64 \mathrm{kpc}, & y=0.05, \beta=0 \\ 43 \mathrm{kpc}, & y=0.01, \beta=0 \\ 88 \mathrm{kpc}, & y=0.05, \beta=0.2 \\ 72 \mathrm{kpc}, & y=0.01, \beta=0.2\end{cases}
$$

By direct fitting of his sample, S95 empirically deduced $R_{*}=$ $55 \mathrm{kpc}$ and inferred $f_{c}=1, \beta=0.2$, and $y=0.05$. Assuming $f_{c}=1, \beta=0.2$, and $y=0.05$, we computed a statistical covering fraction corrected absorber halo radius of $R_{*}=$ $88 \mathrm{kpc}$. The difference between the two values arises from the different methods used to determine $R_{*} ; \mathrm{S} 95$ applied a fit to a known sample of Mg II absorption selected galaxies, whereas our values are directly computed from measured absorption and galaxy statistics. Assuming $f_{c}$ less than unity would increase our computed value of $R_{*}$, yielding a value even less consistent with that of S95.

In Figure 1(b), the projected quasar-galaxy separation, $D$, is plotted versus $L_{B} / L_{B}^{*}$. The mean impact parameter is $\langle D\rangle=$ $53.2 \mathrm{kpc}$ which is close to the S95 halo size. The dash-dot line is the halo radius, $R(L)$, from Equation (1) using $R_{*}=$ $55 \mathrm{kpc}, f_{c}=1$, and $\beta=0.2$ found by S95. Three non-absorbing galaxies reside below the $R(L)$ boundary and five reside above. This is not necessarily inconsistent with S95, who found 2 of 14 non-absorbing galaxies below the $R(L)$ boundary. However, we find $16 W_{r}(2796) \geqslant 0.3 \AA$ absorbers that are outside the
$R(L)$ boundary by as much as $60 \mathrm{kpc}$. In the standard halo model, galaxies above the $R(L)$ boundary are expected not to be associated with $W_{r}(2796) \geqslant 0.3 \AA$ absorption. The dashdash line is the halo radius, $R(L)$, from Equation (1) using the parameters $R_{\mathrm{x}}=88 \mathrm{kpc}, f_{c}=1$, and $\beta=0.2$. We find that five of the eight non-absorbing galaxies lie below the $R(L)$ boundary. These five galaxies are expected to be strong absorbing galaxies if they obey the $R(L)$ relation. Also, there are three absorbing galaxies above the $R(L)$ boundary.

From Figure 1(b), it would appear that the value of $\beta$ is not constrained for the $B$-band luminosities since non-absorbing galaxies are both above and below $R(L)$ for both the $R_{*}=$ $55 \mathrm{kpc}$ deduced by $\mathrm{S} 95$ and our computed size of $R_{\mathrm{x}}=88 \mathrm{kpc}$. Assuming that there is no luminosity scaling, we explore halo cross sections with $\beta=0$. In Figure 2(a), we plot $W_{r}(2796)$ versus $D$. The vertical line is the statistical absorber radius, $R_{\mathrm{x}}=64 \mathrm{kpc}$ (where $D / R_{\mathrm{x}}=1$ ), for $\beta=0$ and $y=0.05$. The top axis gives $D / R_{\mathrm{x}}$. Galaxies to the left of the line are consistent with the computed statistical absorber radius. Galaxies to the right of the line are inconsistent; if the standard halo model applies these particular galaxies must have halos with $f_{c}<1$. We find 5 of 29 galaxies at $D>R_{\mathrm{x}}$. If we assume $y=0.01$ and $\beta=0$, we obtain $R_{\mathrm{x}}=43 \mathrm{kpc}$ and find 16 galaxies reside at $D>R_{\mathrm{x}}$ and that 4 non-absorbing galaxies are expected to have $W_{r}(2796) \geqslant 0.3 \AA$ absorption. Note that $R_{\mathrm{X}}$ is very sensitive to the choice of the luminosity cutoff when $\beta=0$. Larger $\beta$ suppresses the faint-end slope in Equation (2), reducing the cross-sectional contribution of the lowest luminosity galaxies that dominate by number.

In Figure 2(b), we plot $W_{r}(2796)$ versus $D / R(L)$. The dashdash line is $D / R(L)=1$ for $R_{\mathrm{x}}=88 \mathrm{kpc}, \beta=0.2$, and $y=0.05$. Again, three of 29 galaxies have $D / R(L)>1$ and five non-absorbing galaxies have $D / R(L)<1$. If we assume $y=0.01$ and $\beta=0.2$, we obtain $R_{\mathrm{x}}=72 \mathrm{kpc}$, which increments the number of galaxies at $D / R(L)>1$ to 7 . If we apply $\beta=0.28$ from Guillemin $\&$ Bergeron (1997), then $R_{\mathrm{x}}$ increases to $100 \mathrm{kpc}$ (for $y=0.05$ ) and only three absorbing galaxies lie above the $R(L)$ boundary. The dash-dot line is the S95 result where $D / R(L)=1$ for $R_{*}=55 \mathrm{kpc}$. It is clear that there is a significant fraction of absorbing galaxies that is not well represented by the standard halo model of S95, since 

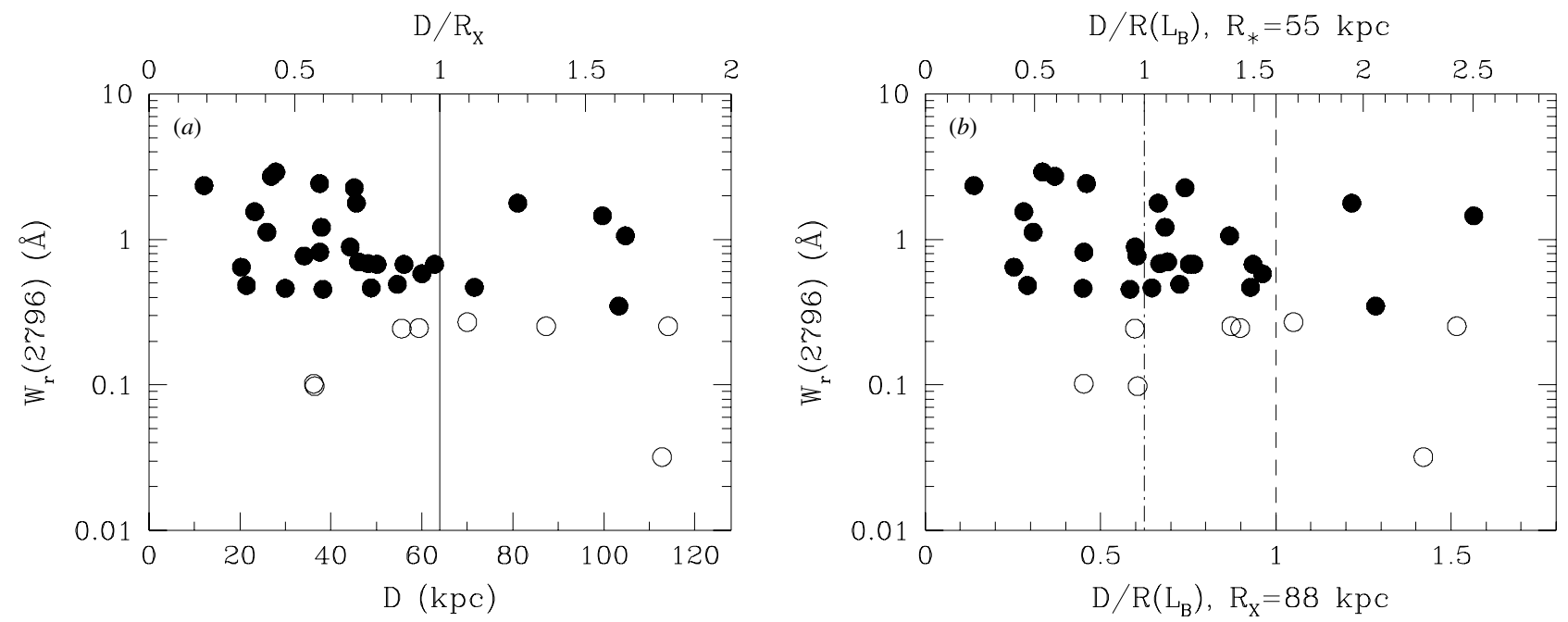

Figure 2. (a) $W_{r}$ (2796) as a function $D$ (bottom axis) and $D / R_{\mathrm{X}}$ (top axis). The solid line represents $R_{\mathrm{x}}=64 \mathrm{kpc}$ for $\beta=0$ and for a luminosity cutoff of $0.05 L_{B}^{*}$. (b) $W_{r}(2796)$ as a function of $D / R\left(L_{B}\right)$. The dashed-dotted line represents $R_{*}=55 \mathrm{kpc}$ and the dash-dash line represents $R_{\mathrm{x}}=88 \mathrm{kpc}$ using $\beta=0.2$ and $y=0.05$.

Table 1

Mg II Halo Gas Covering Fractions

\begin{tabular}{|c|c|c|c|c|c|}
\hline \multirow[b]{2}{*}{ No. ${ }^{a}$} & \multirow[b]{2}{*}{$f_{c}$} & \multicolumn{2}{|c|}{$y=0.05$} & \multicolumn{2}{|c|}{$y=0.01$} \\
\hline & & $\beta=0, R_{\mathrm{x}}=64 \mathrm{kpc}$ & $\beta=0.2, R_{\mathrm{x}}=88 \mathrm{kpc}$ & $\beta=0, R_{\mathrm{x}}=43 \mathrm{kpc}$ & $\beta=0.2, R_{\mathrm{x}}=72 \mathrm{kpc}$ \\
\hline 1. & $w_{L}(\beta, y)\left(105 / R_{\mathrm{X}}\right)^{-2}$ & 0.37 & 0.37 & 0.17 & 0.17 \\
\hline 2. & $\left\langle D / R\left(L_{B}\right)\right\rangle^{-2}$ & 0.52 & 0.79 & 0.58 & 0.63 \\
\hline 3. & $\left(R_{\mathrm{x}} / 55\right)^{-2}$ & 0.74 & 0.40 & $-^{\mathrm{b}}$ & 0.58 \\
\hline & $\left\langle f_{c}\right\rangle$ & 0.54 & 0.52 & 0.38 & 0.46 \\
\hline
\end{tabular}

Notes.

${ }^{a}$ The different methods for computed the covering fractions: (1) the luminosity function weighted $f_{c}$, assuming $R_{*}$ is to be equal to the maximum impact parameter of $D_{\max }=105 \mathrm{kpc}$. (2) The average of the covering fractions for each galaxy was computed for galaxies with impact parameters greater then the statistical halo size. (3) The statistical halo size is assumed to be $55 \mathrm{kpc}(\mathrm{S} 95)$.

${ }^{\mathrm{b}}$ Our sample of galaxies provide no constraint on the covering fraction for $y=0.01$ and $\beta=0$.

it is expected that spherically symmetric gas halos with unity covering fraction would give rise to absorption exclusively at $D / R(L) \leqslant 1$

\section{DISCUSSION}

Our sample of galaxies is not statistically complete, due to the chosen method of searching for galaxies selected by $\mathrm{Mg}$ II absorption (see Churchill et al. 2005). None the less, the data clearly support a covering fraction less than unity, based upon the deduced statistical absorber radius, $R_{\mathrm{x}}$, which is computed from the redshift path density of the full population of $\mathrm{Mg}$ II absorbers.

As seen in Figures 2(a) and (b), a substantial fraction of galaxies are found at impact parameters well beyond the statistical absorber radius. The largest impact parameter in our sample is $D_{\max } \simeq 105 \mathrm{kpc}$. If we assume that the largest impact parameter is a proxy for the true size of the covering fraction corrected absorbing halo radius such that $R_{*}=D_{\max }$, then we can compute a luminosity function weighted covering fraction where $f_{c}=w_{L}(\beta, y)\left(105 / R_{\mathrm{x}}\right)^{-2}$. Assuming a lower galaxy luminosity cutoff of $y=0.05$, we obtain $f_{c}=0.37$ for $\beta=0$ and $f_{c}=0.37$ for $\beta=0.2$. Assuming a lower luminosity cutoff of $y=0.01$, we obtain $f_{c}=0.17$ for $\beta=0$ and $f_{c}=0.17$ for $\beta=0.2$. These results are summarized in Table 1 . Now with $f_{c}<1$, the presence of eight non-absorbing galaxies within the statistical halo radius, $R(L)$, is consistent with $R_{*} \simeq 105 \mathrm{kpc}$ for $W_{r}(2796) \geqslant 0.3 \AA$.

Using each galaxy from our sample, a conservative estimate of the covering fraction is the mean of the upper limit on $f_{c}=(D / R(L))^{-2}$. In a complete sample, each galaxy with $D>R(L)$ makes a fractional contribution to reducing the gas covering fraction. Galaxies with $D \leqslant R(L)$ provide no constraint. If our sample is representative of a complete sample, we obtain $\left\langle f_{c}\right\rangle=0.52(y=0.05, \beta=0),\left\langle f_{c}\right\rangle=0.79$ $(y=0.05, \beta=0.2),\left\langle f_{c}\right\rangle=0.58(y=0.01, \beta=0)$, and $\left\langle f_{c}\right\rangle=0.63(y=0.01, \beta=0.2)$.

If we assume that $R_{*}=55 \mathrm{kpc}$ of $\mathrm{S} 95$ is the true halo size, we can also compute the covering fractions such that $f_{c}=\left(R_{\mathrm{x}} / R_{*}\right)^{-2}$. We obtain $f_{c}=0.40(y=0.05, \beta=0.2)$, $f_{c}=0.58,(y=0.01, \beta=0.2)$, and $f_{c}=0.74(y=0.05, \beta=$ $0) . R_{*}>R_{\mathrm{x}}$ for $y=0.01, \beta=0$ yields no constraint on $f_{c}$. The results of the above computations of the covering fractions are summarized in Table 1.

From all the methods of estimating $f_{c}$, we obtain $\left\langle f_{c}\right\rangle \sim 0.5$ with a range of $0.17 \leqslant f_{c} \leqslant 0.80$. This is consistent with $f_{c}=0.7-0.8$ deduced by Charlton \& Churchill (1996) from Monte Carlo simulations of $\mathrm{Mg}$ II absorption selected galaxy surveys. Our average $f_{c}$ also consistent with the result of Tripp 
\& Bowen $(2005)^{6}$ who find $f_{c} \sim 0.55$ and higher than $f_{c} \sim 0.25$ determined by Bechtold \& Ellingson (1992). Also, Churchill et al. (2007) found a galaxy, probed well within the $R(L)$ boundary, that exhibits no $\mathrm{Mg}$ II absorption to $W_{r}(2796) \leqslant$ $7 \mathrm{m \AA}$. All these results suggest $f_{c}<1$ for Mg II absorbing gas with $W_{r}(2796) \geqslant 0.3 \AA$. Thus, non-absorbing galaxies below the predicted halo size are expected.

Although the data do not clearly support a halo sizeluminosity scaling, if we apply $f_{c} \sim 0.5$ such that the covering fraction corrected absorbing halo radius is $R_{*}=1.41 R_{\mathrm{x}}$, a Holmberg-like luminosity relationship with $\beta \simeq 0.2$ is not ruled out for both $y=0.05$ and $y=0.01$. We can further constrain $f_{c}, R_{*}$, and $\beta$ with a maximum likelihood fit that satisfies the distribution of impact parameters and luminosities of our sample. In this analysis, $R_{*}=\sqrt{f_{c}} R_{\mathrm{x}}$ is a function of $\beta$ as constrained by $d N / d z$. First, we assume that all absorbing galaxies must reside below the $R(L)$ boundary. For $y=0.05$, we find an upper limit of $f_{c} \leqslant 0.4$ for a range of $0.02 \leqslant \beta \leqslant 0.24$ with $105 \leqslant R_{*} \leqslant 150 \mathrm{kpc}$, respectively. For lower covering fractions, the allowed ranges of $\beta$ and $R_{*}$ increase. For $y=0.01$, we find $f_{c} \leqslant 0.2$ for $0.04 \leqslant \beta \leqslant 0.66$ with $110 \leqslant R_{*} \leqslant$ $290 \mathrm{kpc}$, respectively. If we relax the condition such that one to three absorption selected galaxies may reside above the $R(L)$ boundary, which could account for errors in the luminosities and/or our finite sample, then the allowed ranges of $f_{c}, R_{*}$, and $\beta$ increase. For these cases with $y=0.05$, we find an upper limit of $f_{c} \leqslant 0.7$ for $0.18 \leqslant \beta \leqslant 0.58$ with $80 \leqslant R_{*} \leqslant 150 \mathrm{kpc}$, respectively. Thus, our sample is consistent with a Holmberglike luminosity relationship in the case $f_{c} \lesssim 0.5$.

A central issue to this discussion is whether there is a fundamental physical difference between the halos of non-absorbing (weak) and absorbing galaxies or whether the difference in $W_{r}(2796)$ arises only from a chance intersection of the quasar line of sight through a single gas cloud or a gas cloud complex in these halos. Even if weak $W_{r}(2796)<0.3 \AA$ systems are similar to strong systems, and differ only by the number of clouds intersected along the quasar line of sight, our arguments for constraining the halo gas covering fraction for $W_{r}(2796) \geqslant$ $0.3 \AA$ still hold.

Strong absorbers are typically characterized by a dominant and blended subsystem and accompanied by significantly weaker subsystems at relative velocities ranging from $\sim 40$ to $100 \mathrm{~km} \mathrm{~s}^{-1}$ (Churchill \& Vogt 2001). In fact, there may be different physical processes governing the $W_{r}$ (2796) distribution of weak absorption associated with strong absorbers and the general population of weak absorbers. Churchill et al. (1999) determined that the number density of the general population of weak systems increases as $W_{r}(2796)$ decreases down to $0.02 \AA$. In contrast, the Mg II equivalent width distribution of intermediate- and high-velocity subsystems in strong absorbers turns over below $W_{r}(2796) \sim 0.08 \AA$ (Churchill \& Vogt 2001; Mshar et al. 2007). These facts suggest that lines of sight through galaxy halos often probe a dominant, more massive structure surrounded by smaller fragments of gas; a scenario consistent with patchy halos, in which some lines of sight near galaxies would be expected to probe only $W_{r}(2796) \gtrsim 0.08 \AA$ weak absorption.

\footnotetext{
6 Tripp \& Bowen (2005) have a $W_{r}(2796) \sim 0.1 \AA(2 \sigma)$ detection limit which translates to a $W_{r}(2796) \sim 0.25 \AA(5 \sigma)$ detection limit as presented here. We have removed all absorbers with $W_{r}(2796)<0.25 \AA$ in order to compare our results at the same detection limit.
}

There is also the possibility that some of the galaxies in our sample having redshifts consistent with those of the $\mathrm{Mg}$ II absorbers may not be the primary structure responsible for the absorption. In some cases there could be a faint unidentified galaxy located directly in front of the quasar that cannot be identified even with careful point-spread subtraction of the quasar (see Steidel et al. 1997). Thus, our estimated values of $f_{c}$ and $R_{*}$ could be slightly skewed toward smaller and larger values, respectively. It is difficult to quantify this effect since such putative faint galaxies could actually be companions to the galaxies in our sample.

\section{CONCLUSIONS}

In conclusion, the gas covering fraction must be less than unity since the observed impact parameter distribution of absorbing galaxies does not fall exclusively within the statistical absorber halo radius in the range of $43 \leqslant R_{\mathrm{x}} \leqslant 88 \mathrm{kpc}$. The fact that some absorbing galaxies are found at $D>R_{\mathrm{x}}$ and some non-absorbing galaxies are found at $D<R_{\mathrm{x}}$ implies $f_{c}<1$ and that the standard halo model cannot describe halos on a caseby-case basis. This highlights the power of using the statistics of absorption line surveys to constrain the properties of halos in relation to the measured distributions in absorption selected galaxy surveys.

By quantifying how individual galaxy halos deviate from a "standard" halo, we have obtained an average gas covering fraction of $\left\langle f_{c}\right\rangle \sim 0.5$. It is possible that $f_{c}$ exhibits both a radial and an equivalent width dependence, though we cannot address this with our sample. Values of $f_{c}$ are likely to depend on galaxy star formation rates, and galaxy-galaxy mergers and harassment histories; processes that give rise to patchy and geometrically asymmetric gas distributions. Alternatively, the absorption properties of intermediate redshift halos may be governed by the dark matter over density, $\Delta \rho / \rho$, and redshifts at which the galaxies formed (Churchill et al. 2007).

Our results also show that, if $f_{c}<1$, the sizes of $\mathrm{Mg}$ II absorbing halos can still follow a Holmberg-like luminosity relation with $\beta$ in the range of $0.2-0.28$ (S95; Guillemin \& Bergeron 1997), which corresponds to $R_{*} \sim 110 \mathrm{kpc}$. If $\beta=0$ is assumed, then $f_{c} \leqslant 0.37$ for our sample to be consistent with no luminosity scaling. In semi-analytical models in which $\mathrm{Mg}$ II absorbing gas is infalling and is pressure confined within the cooling radius of hot halos (e.g., Mo \& Miralda-Escude 1996; Burkert \& Lin 2000; Lin \& Murray 2000; Maller \& Bullock 2004), a Holmberg-like luminosity relation in quasar absorption line systems naturally arises (Mo \& Miralda-Escude 1996). However, these models have great difficulty explaining $\mathrm{Mg}$ II absorption at impact parameters greater than $\sim 70 \mathrm{kpc}$. If on the other hand halo gas spatial distributions are governed by stochastic mechanical processes, as suggested by Kacprzak et al. (2007a), then there is no a priori reason to expect a clean halo-size luminosity scaling. It is likely that some combination of these scenarios contribute to the statistical values of $f_{c}$ and $\beta$. Thus, it is reasonable to suggest that $\mathrm{Mg}$ II halo sizes may not be strictly coupled to the host galaxy luminosity.

Further work on the cross-correlations between absorbers and galaxies would provide better estimates of $f_{c}$ and $\beta$, two quantities that provide direct constraints of galaxy formation simulations. Also needed are additional constrains on the relative kinematics of the absorbing halo gas and galaxies (e.g., Steidel et al. 2002; Ellison et al. 2003; Kacprzak et al. $2007 \mathrm{~b}$ ). What is required is the development of techniques to quantitatively compare observational data with mock quasar 
absorption line analysis of simulated galaxy halos (Churchill et al. 2006).

Partial support from program no. 10644 which was provided by NASA through a grant from the Space Telescope Science Institute. Partial support for G.G.K was also provided by SigmaXi Grants in Aid of Research. G.G.K thanks NMSU for funding from the Graduate Student Enhancement Grant. M.T.M. thanks PPARC for and Advanced Fellowship. We thank Hsiao-Wen Chen for discussions regarding k-corrections. We also thank the anonymous referee for insightful comments. Some of the data presented herein are based on observations made with the NASA/ESA Hubble Space Telescope, obtained from the data archive at the Space Telescope Institute. STScI is operated by the association of Universities for Research in Astronomy, Inc. under the NASA contract NAS 5-26555. Some spectroscopic data were obtained at the W. M. Keck Observatory, which is operated as a scientific partnership among the California Institute of Technology, the University of California and NASA. The Observatory was made possible by the generous financial support of the W. M. Keck Foundation. Additional spectroscopic data are based on observations made with European Southern Observatory Very Large Telescope at the Paranal Observatories under various programs.

Facilities: HST (WFPC-2), Keck I (HIRES), VLT (UVES).

\section{REFERENCES}

Bechtold, J., \& Ellingson, E. 1992, ApJ, 396, 20

Bergeron, J., \& Boissé, P. 1991, A\&A, 243, 334

Bowen, D. V., Blades, J. C., \& Pettini, M. 1995, ApJ, 448, 634

Burkert, A., \& Lin, D. N. C. 2000, ApJ, 537, 270

Charlton, J. C., \& Churchill, C. W. 1996, ApJ, 465, 631

Churchill, C. W., Kacprzak, G., Ceverino, D., Evans, J., \& Widhalm, A. 2006 BAAS, 38, 998

Churchill, C. W., Kacprzak, G. G., \& Steidel, C. C. 2005, in IAU 199 Proc., Probing Galaxies through Quasar Absorption Lines, ed. P. R. Williams, C.-G. Shu, \& B. Ménard (Cambridge: Cambridge Univ. Press), 24

Churchill, C. W., Kacprzak, G. G., Steidel, C. C., \& Evans, J. L. 2007, ApJ, 661,714
Churchill, C. W., Rigby, J. R., Charlton, J. C., \& Vogt, S. S. 1999, ApJS, 120,51

Churchill, C. W., \& Vogt, S. S. 2001, AJ, 122, 679

Dekker, H., D'Odorico, S., Kaufer, A., Delabre, B., \& Kotzlowski, H. 2000, SPIE, 4008, 534

Drory, N., Bender, R., Feulner, G., Hopp, U., Maraston, C., Snigula, J., \& Hill, G. J. 2003, ApJ, 595, 698

Ellison, S. L., Mallén-Ornelas, G., \& Sawicki, M. 2003, ApJ, 589, 709

Faber, S. M., et al. 2007, ApJ, 665, 265

Fukugita, M., Shimasaku, K., \& Ichikawa, T. 1995, PASP, 107, 945

Guillemin, p., \& Bergeron, J. 1997, A\&A, 328, 499

Kacprzak, G. G., Churchill, C. W., Steidel, C. C., Murphy, M. T., \& Evans, J. L. 2007a, ApJ, 662, 909

Kacprzak, G. G., Churchill, C. W., Steidel, C. C., Ceverino, D., Klypin, A. A., \& Murphy, M. T. 2007b, in ASP Conf. Proc., Formation and Evolution of Galaxy Disks, eds. J. G Funes, S. J., \& E. M. Corsini, (San Francisco, CA: Astronomical Society of the Pacific), arXiv:0710.4962

Kaufmann, T., Mayer, L., Wadsley, J., Stadel, J., \& Moore, B. 2006, MNRAS, 370,1612

Kim, A., Goobar, A., \& Perlmutter, S. 1996, PASP, 108, 190

Kinney, A. L., Calzetti, D., Bohlin, R. C., McQuade, K., Storchi-Bergmann, T., \& Schmitt, H. R. 1996, ApJ, 467, 38

Lanzetta, K. M., Bowen, D. V., Tytler, D., \& Webb, J. K. 1995, ApJ, 442,538

Lin, D. N. C., \& Murray, S. D. 2000, ApJ, 540, 170

Lin, W.-P., \& Zou, Z.-L. 2001, ChJAA, 1, 21

Maller, A. H., \& Bullock, J. S. 2004, MNRAS, 355, 694

Mo, H. J., \& Mao, S. 2002, MNRAS, 333, 768

Mo, H. J., \& Miralda-Escude, J. 1996, ApJ, 469, 589

Mshar, A. C., Charlton, J. C., Lynch, R. S., Churchill, C., \& Kim, T.-S. 2007 arXiv:0706.0515

Nestor, D. B., Turnshek, D. A., \& Rao, S. M. 2005, ApJ, 628, 637

Steidel, C. C. 1995, in QSO Absorption Lines, ed. G. Meylan (Berlin: Springer) 139

Steidel, C. C., Dickinson, M., Meyer, D. M., Adelberger, K. L., \& Sembach, K. R. 1997, ApJ, 480, 586

Steidel, C. C., Dickinson, M., \& Persson, S. E. 1994, ApJ, 437, L75

Steidel, C. C., Kollmeier, J. A., Shapley, A. E., Churchill, C. W., Dickinson, M., \& Pettini, M. 2002, ApJ, 570, 526

Tripp, T. M., \& Bowen, D. V. 2005, in IAU 199 Proc., Probing Galaxies through Quasar Absorption Lines, ed. P. R. Williams, C.-G. Shu, \& B. Ménard (Cambridge, MA: Cambridge Univ. Press), 5

Vogt, S. S., et al. 1994, SPIE, 2198, 362

White, S. D. M., \& Rees, M. J. 1978, MNRAS, 183, 341

Zibetti, S., Ménard, B., Nestor, D. B., Quider, A. M., Rao, S. M., \& Turnshek, D. A. 2007, ApJ, 658, 161 